\title{
Transition to the Ultimate Regime in Two-Dimensional Rayleigh-Bénard Convection
}

\author{
Xiaojue Zhu, ${ }^{1, *}$ Varghese Mathai, ${ }^{1}$ Richard J. A. M. Stevens, ${ }^{1}$ Roberto Verzicco, ${ }^{2,1}$ and Detlef Lohse ${ }^{1,3, \dagger}$ \\ ${ }^{1}$ Physics of Fluids Group and Max Planck Center Twente for Complex Fluid Dynamics, \\ MESA+Institute and J. M. Burgers Centre for Fluid Dynamics, University of Twente, \\ P.O. Box 217, 7500AE Enschede, The Netherlands \\ ${ }^{2}$ Dipartimento di Ingegneria Industriale, University of Rome "Tor Vergata", Via del Politecnico 1, Roma 00133, Italy \\ ${ }^{3}$ Max Planck Institute for Dynamics and Self-Organization, 37077 Göttingen, Germany
}

(Received 4 January 2018; published 6 April 2018)

\begin{abstract}
The possible transition to the so-called ultimate regime, wherein both the bulk and the boundary layers are turbulent, has been an outstanding issue in thermal convection, since the seminal work by Kraichnan [Phys. Fluids 5, 1374 (1962)]. Yet, when this transition takes place and how the local flow induces it is not fully understood. Here, by performing two-dimensional simulations of Rayleigh-Bénard turbulence covering six decades in Rayleigh number Ra up to $10^{14}$ for Prandtl number $\operatorname{Pr}=1$, for the first time in numerical simulations we find the transition to the ultimate regime, namely, at $\mathrm{Ra}^{*}=10^{13}$. We reveal how the emission of thermal plumes enhances the global heat transport, leading to a steeper increase of the Nusselt number than the classical Malkus scaling $\mathrm{Nu} \sim \mathrm{Ra}^{1 / 3}$ [Proc. R. Soc. A 225, 196 (1954)]. Beyond the transition, the mean velocity profiles are logarithmic throughout, indicating turbulent boundary layers. In contrast, the temperature profiles are only locally logarithmic, namely, within the regions where plumes are emitted, and where the local Nusselt number has an effective scaling $\mathrm{Nu} \sim \mathrm{Ra}^{0.38}$, corresponding to the effective scaling in the ultimate regime.
\end{abstract}

DOI: $10.1103 /$ PhysRevLett.120.144502

Rayleigh-Bénard (RB) flow, in which the fluid is heated from below and cooled from above, is a paradigmatic representation of thermal convection, with many features that are of interest in natural and engineering applications [1-3]. When the temperature difference between the two plates (expressed as the dimensionless parameter Rayleigh number Ra) is large enough, the system is expected to undergo a transition from the so-called "classical regime" of turbulence, where the boundary layers (BLs) are of the laminar type [4-7], to the so-called "ultimate regime," where the BLs are of the turbulent type, as first predicted by Kraichnan [8] and later by others [9-13]. In the classical regime, the Nusselt number $\mathrm{Nu}$ (dimensionless heat transfer) is known to effectively scale as $\mathrm{Ra}^{\beta}$, with the effective scaling exponent $\beta \leq 1 / 3[10,11,14-16]$. Beyond the transition to the ultimate regime, the heat transport is expected to increase substantially, reflected in an effective scaling exponent $\beta>1 / 3[1,8,12]$.

Hitherto, the evidence for the transition to the ultimate regime has come only from experimental measurements of $\mathrm{Nu}$. In fact, the community is debating at what $\mathrm{Ra}$ the transition starts and even whether there is a transition at all. For example, Niemela and Sreenivasan [17] observed that $\beta$ first increases above $1 / 3$ around $\mathrm{Ra} \approx 10^{14}$ and then decreases back to $1 / 3$ again for $\mathrm{Ra} \approx 10^{15}$. Subsequently, Urban et al. [18] also reported $\beta \approx 1 / 3$ for $\mathrm{Ra}=\left[10^{12}, 10^{15}\right]$. However, Chavanne et al. $[19,20]$ found that the effective scaling exponent $\beta$ increases to 0.38 for $\mathrm{Ra}>2 \times 10^{11}$. In the experiments mentioned above, low-temperature helium was used as the working fluid, and Prandtl number $\mathrm{Pr}$ changes with increasing $\mathrm{Ra}$. In contrast to helium, $\mathrm{SF}_{6}$ has roughly pressure independent $\mathrm{Pr}$. This allowed $\mathrm{He}$ et al. $[21,22]$ to achieve the ultimate regime more conclusively. They observed a similar exponent 0.38 , but this exponent was found to start only at a much higher $\mathrm{Ra} \approx 10^{14}$ (the transition starts at $\mathrm{Ra} \approx 10^{13}$ ). This observation is compatible with the theoretical prediction $[10,11]$ for the onset of the ultimate regime. It is also consistent with the theoretical prediction of Refs. [8,12], according to which a logarithmic temperature and velocity BLs are necessary to obtain an effective scaling exponent $\beta \approx 0.38$ for that Ra.

The apparent discrepancies among various high-Ra RB experiments have been attributed to many factors. The change of Pr, the non-Boussinesq effect, the use of a constant temperature or constant heat flux condition, the finite conductivity of the plates, and the sidewall effect can all play different roles $[1,23]$. Direct numerical simulations (DNS), which do not have these unavoidable artifacts as occurring in experiments, can ideally help to understand the transition to the ultimate regime, with the strict accordance to the intended theoretic RB formulations. Unfortunately, high$\mathrm{Ra}$ simulations in three dimensions (3D) are prohibitively expensive [24,25]. The highest Rayleigh number achieved in 3D RB simulations is $2 \times 10^{12}$ [23], which is one order of 
magnitude short of the expected transitional Ra. Twodimensional (2D) RB simulations, though different from 3D ones in terms of integral quantities for small $\operatorname{Pr}[26,27]$, still capture many essential features of 3D RB [27]. Consequently, in recent years, 2D DNS has been widely used to test theories, not only for normal RB $[28,29]$ but also for RB in porous media [30]. Although also expensive at high Ra, now we have the chance to push forward to $\mathrm{Ra}=10^{14}$ using $2 \mathrm{D}$ simulations as we will show in this Letter.

Another advantage of DNS is that velocity and temperature profiles can be easily measured, to check whether they are logarithmic in the ultimate regime, as expected from the theory. Specifically, for the temperature, only a few local experimental measurements were available in the nearsidewall regions of RB cells, which showed logarithmic profiles $[31,32]$. Even worse, for the velocity, there is almost no evidence for the existence of a logarithmic BL, due to the experimental challenges. For instance, in cylindrical cells with aspect ratio $\Gamma=\mathcal{O}(1)$, the mean velocity profile cannot be easily quantified because of the absence of a stable mean roll structure [24]. In situations where stable rolls do exist (e.g., narrow rectangular cells), the highest $\mathrm{Ra}$ available are still far below the critical $\mathrm{Ra}$ at which logarithmic velocity BLs can manifest themselves [4,7].

As DNS provides us with every detail of the flow field which might be unavailable in experiments, it also enables us to reveal the links between the global heat transport and the local flow structures. A few attempts (both 2D and 3D) have been made in the classical regime, in which logarithmic temperature BLs were detected, by selectively sampling the regions where the plumes are ejected to the bulk $[31,33]$. However, it is still unclear how these local logarithmic BLs contribute to the attainment of the global heat transport enhancement during the transition to the ultimate regime.

In this work, we observe the transition to the ultimate regime in 2D for the first time in DNS, namely, at $\mathrm{Ra}^{*}=10^{13}$, similar as in the $3 \mathrm{D} \mathrm{RB}$ experiments of Ref. [21]. DNS also provides the first evidence that the mean velocity profiles follow the log law of the wall, in analogy to other paradigmatic turbulent flows [34-36]. Furthermore, we explore the link between the local and global quantities to reveal the mechanism leading to the increased scaling exponent beyond the transition.

The simulations have been carried out using a wellvalidated second-order finite-difference code [37,38]. The two control parameters are $\mathrm{Ra}=\alpha g \Delta L^{3} /(\nu \kappa)$ and $\operatorname{Pr}=\nu / \kappa$, with $\alpha$ being the thermal expansion coefficient, $g$ the gravitational acceleration, $\Delta$ the temperature difference across a fluid layer of depth $L, \nu$ the kinematic viscosity, and $\kappa$ the thermal diffusivity. In the simulations, $\operatorname{Pr}$ is fixed at 1 and aspect ratio $\Gamma \equiv W / L$ is fixed at 2 , where $W$ is the width of the domain. With this $\Gamma$, it has been found that the heat flux approximates the heat flux at an infinite aspect ratio [39]. The boundary conditions are no-slip for the velocity, a constant temperature for the bottom and top plates, and periodic horizontally. $\mathrm{Nu}$ is calculated from the relation $\mathrm{Nu}=\sqrt{\operatorname{Ra} \operatorname{Pr}}\left\langle u_{z} \theta\right\rangle_{A, t}-\left\langle\partial_{z} \theta\right\rangle_{A, t}$, with $u_{z}$ being the vertical velocity, $\theta$ the temperature, and $\langle\ldots\rangle_{A, t}$ the average over a horizontal plane and time. All the cases were well resolved. At the highest $\mathrm{Ra}=10^{14}$, we used a grid with $20480 \times 10240$ mesh points. For details of the simulations, we refer to the Supplemental Material [40].

We begin by looking at the heat transport as a function of Ra. In Fig. 1, we show $\mathrm{Nu}(\mathrm{Ra})$ compensated with $\mathrm{Ra}^{0.35}$, for the range $\mathrm{Ra}=\left[10^{8}, 10^{14}\right]$. Up to $\mathrm{Ra}=10^{11}$ (blue symbol), the effective scaling is essentially the same $(\beta \approx 0.29)$ as has been already observed $[27,39,41]$ in the classical regime where the BLs are laminar $[5,6]$. This trend continues up to the transitional Rayleigh number $\mathrm{Ra}^{*}=10^{13}$ (green symbol). Beyond this, we witness the start of the transition to the ultimate regime, with a notably larger effective scaling exponent $\beta \approx 0.35$, as evident from the plateau in the compensated plot.

Next, to appreciate how the flow structures are different before and beyond the transition $\left(\mathrm{Ra}^{*}\right)$, we show the respective instantaneous temperature fields, see Fig. 2. The top panel presents a relatively low $\mathrm{Ra}=10^{11}$ (below $\left.\mathrm{Ra}^{*}\right)$, while the middle panel shows a high $\mathrm{Ra}=10^{14}$ (beyond $\mathrm{Ra}^{*}$ ). At low Ra, intense large scale rolls (LSRs) are clearly visible. In comparison, at high Ra, the LSR, although still evident, contains much weaker and smaller structures. Interestingly, even at the highest Ra, the temperature field still has both plume-ejecting and -impacting regions. Additionally, these observations indicate that the spatial extent of plume-ejecting regions do not grow in spite of the increase in $\mathrm{Ra}$.

We now focus on the mean (space and time) temperature and velocity fields at the transitional Ra. Remarkably, even after 500 dimensionless time units, the flow domain still shows a stable mean roll structure; i.e., the rolls are pinned with clearly demarcated plume-ejecting and -impacting

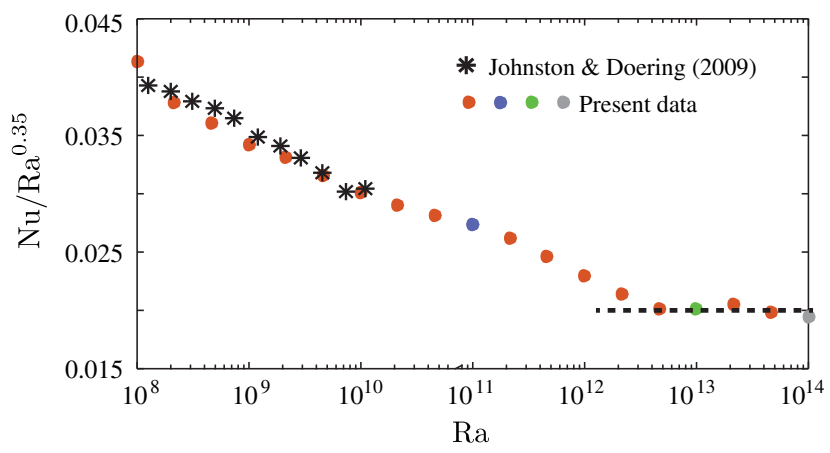

FIG. 1. $\mathrm{Nu}(\mathrm{Ra})$ plot compensated by $\mathrm{Ra}^{0.35}$. The data agree well with the previous results in the low-Ra regime [39]. The flow structures of the three colored data points (blue for $\mathrm{Ra}=10^{11}$, green for $\mathrm{Ra}=10^{13}$, and gray for $\mathrm{Ra}=10^{14}$ ) are displayed in Fig. 2. 


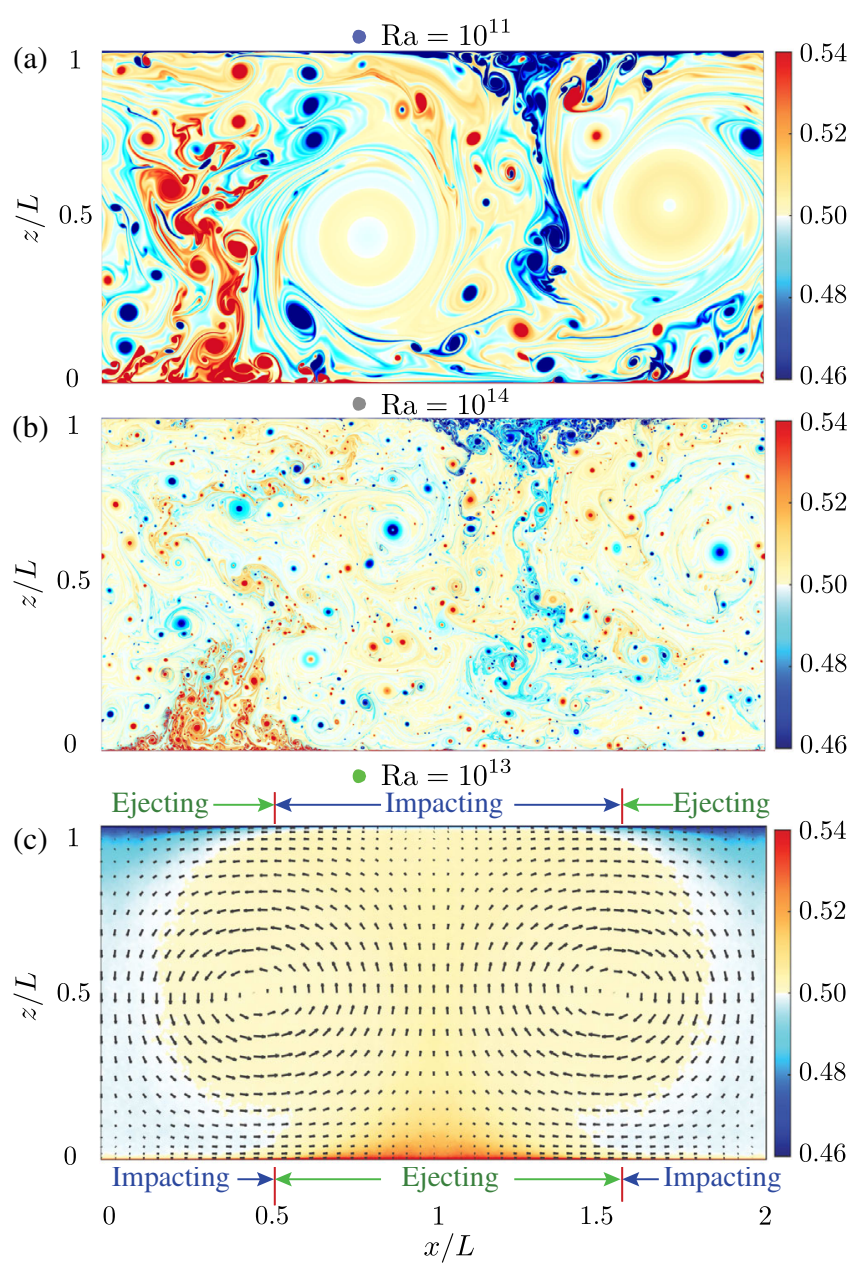

FIG. 2. The instantaneous temperature fields for (a) $\mathrm{Ra}=10^{11}$ and (b) $\mathrm{Ra}=10^{14}$. The corresponding movies are shown in the Supplemental Material [40]. (c) The mean temperature and velocity field for $\mathrm{Ra}=10^{13}$. The contours represent the mean temperature field, while the vectors show the direction of the velocity, scaled by its magnitude. The plate surfaces have been divided into equal-sized plume-ejecting and -impacting regions.

regions [see Fig. 2(c)]. The mean temperature and velocity fields display horizontal symmetry, which enables us to average them over a single LSR instead of the whole domain (as the velocity averaged horizontally for the whole domain will be zero).

Figure 3(a) shows the temporally and spatially averaged velocity and temperature profiles, performed on one single LSR, for the following defined parameters: $u_{\tau}, u^{+},\langle u\rangle_{x, t}$, $T_{\tau}, T^{+},\langle T\rangle_{x, t}$, and $y^{+}$. The velocity profiles are nondimensionalized in terms of $u^{+}$and $y^{+}$, in wall units, where $u^{+}=\langle u\rangle_{x, t} / u_{\tau}$ and $y^{+}=z u_{\tau} / \nu$. Here $u_{\tau}$ is the friction velocity $u_{\tau}=\sqrt{\left.\nu \partial_{z}\langle u\rangle_{x, t}\right|_{z=0}}$ [42]. Similar to channel, pipe, and boundary layer flows, we can identify two distinct layers: a viscous sublayer, where $u^{+}=y^{+}$, followed by a logarithmic region, where the velocity profile follows $u^{+}=\left(1 / \kappa_{v}\right) \ln y^{+}+B_{v}$ [42]. The inverse slope
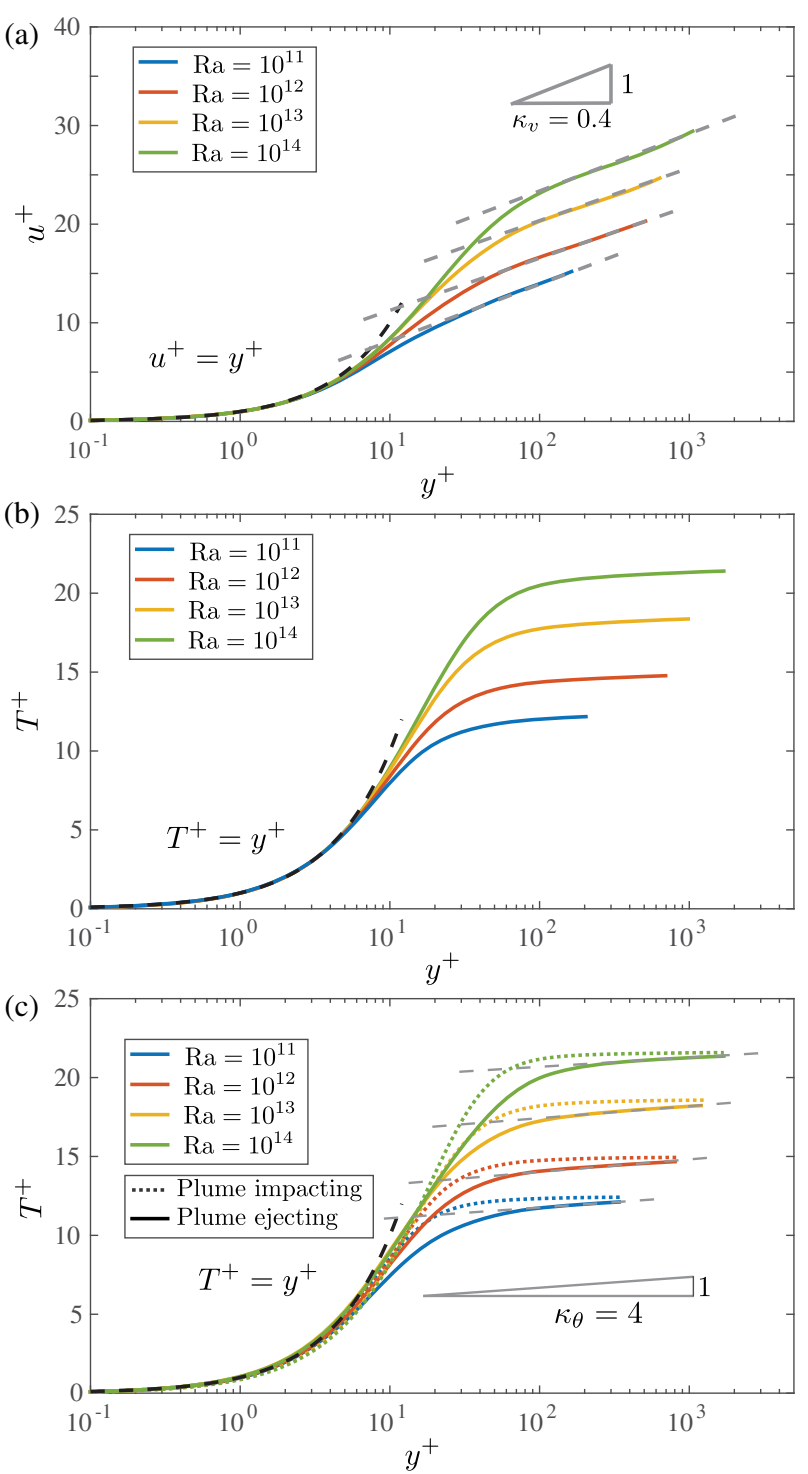

FIG. 3. Mean velocity (a) and temperature (b) profiles in wall units $\left(u^{+}\right.$for velocity, $T^{+}$for temperature, and $y^{+}$for wall distance) at four Ra. The dashed lines show the viscous sublayer behavior and the log-layer behavior. A log layer is seen for the velocity (with inverse slope $\kappa_{v}=0.4$ ) but not for the temperature. (c) Local temperature profiles averaged in plume-ejecting and -impacting regions [see Fig. 2(c) for definitions]. The dashed lines again show the viscous sublayer behavior and the log-layer behavior. A log layer is seen for the temperature in the plumeejecting regions (with inverse slope $\kappa_{\theta}=4.0$ ) but not in impacting regions.

gives $\kappa_{v}=0.4$, which is remarkably close to the von Kármán constant in various 3D canonical wall-bounded turbulent flows [35,36]. However, the parameter $B_{v}$ varies with $\mathrm{Ra}$. With increasing $\mathrm{Ra}$, the logarithmic range grows in spatial extent, until, at $\mathrm{Ra}^{*}=10^{13}$, it spans one decade in $y^{+}$. We then express the averaged temperature profile [Fig. 3(b)] $T^{+}=\left(T_{b}-\langle T\rangle_{x, t}\right) / T_{\tau}$ in wall units, where $T_{b}$ is 
the bottom plate temperature and $T_{\tau}=-\left.\kappa \partial_{z}\langle T\rangle_{x, t}\right|_{z=0} / u_{\tau}$ a characteristic temperature scale analogous to $u_{\tau}$ for the velocity [43]. The mean temperature profile shows a similar viscous sublayer $T^{+}=y^{+}$, followed by a rather flat region, without a clear logarithmic dependence. Since the ultimate regime is associated with logarithmic profiles, the key question remains, as to why the mean temperature profile is not logarithmic despite the global scaling relations suggesting a transition in Fig. 1.

To find out, we look back more closely into the flow field of Fig. 2(c) where the mean flow was separated into (a) a plumeejection region and (b) a plume-impacting region. As noted earlier, the spatial extent of these regions does not grow with increasing $\mathrm{Ra}$, and the mean flow field is horizontally symmetric. Therefore, the domain can be divided into plume-ejection and -impacting regions, enabling us to perform a conditional analysis for the temperature profiles specific to the respective regions. In Fig. 3(c), we plot these profiles separately, for different Ra. Here, the averages are performed based on the regions definition in Fig. 2(c), for $T_{\tau}$, $T^{+},\langle T\rangle_{x, t}, y^{+}$. All the profiles collapse into a single curve in the viscous sublayer. Beyond the viscous sublayer, the impacting and ejecting regions show very different behavior. For the impacting regions, the temperature profile is flat (dotted curves) and remains so for all Ra. However, for the plume-ejecting regions, we observe a clear log layer (solid curves) with a profile $T^{+}=\left(1 / \kappa_{\theta}\right) \ln y^{+}+B_{\theta}$, where $\kappa_{\theta}=4$ is the equivalent von Kármán constant for the temperature and $B_{\theta}$ varies with $\mathrm{Ra}$. Similar to the velocity profiles, the extent of the log layer increases with $\mathrm{Ra}$. At the transitional $\mathrm{Ra}^{*}=10^{13}$, it spans one decade in $y^{+}$.

Temperature profiles that are locally logarithmic (in plume-ejecting regions) have been observed before for both the classical and the ultimate regimes [31-33]. Based on this, one hypothesis regarding how the system undergoes the transition to the ultimate regime is that the fraction of plume-emitting regions (or hot spots) will gradually grow with increasing $\mathrm{Ra}$ [33]. As speculated, the trend would continue until the entire BL becomes a hot spot, thus leading to a mean logarithmic temperature profile. Our findings indicate that here this is not the case, as even at $\mathrm{Ra}=10^{14}$ plume-impacting regions do not show a logarithmic temperature profile. The presence of these impacting regions makes the mean temperature profile also nonlogarithmic [see Fig. 3(b)].

We now explain how the global heat transport scaling can still undergo a transition to the ultimate regime, though only the local temperature profile is logarithmic, not the globally averaged one. We recall that, by definition on the plate surface, $\mathrm{Nu}=-\left\langle\partial_{z} \theta\right\rangle_{A}$. Following the observations from Fig. 2(c), we compute the local $\mathrm{Nu}$ on the plate surface from ejecting $\left(\mathrm{Nu}_{e}\right)$ and impacting $\left(\mathrm{Nu}_{i}\right)$ regions separately. These are shown in Fig. 4, compensated by $\mathrm{Ra}^{1 / 3}$. Up to $\mathrm{Ra}^{*}$, both $\mathrm{Nu}_{i}$ and $\mathrm{Nu}_{e}$ follow a similar trend, with their respective local scaling exponents $\beta_{i}$ and

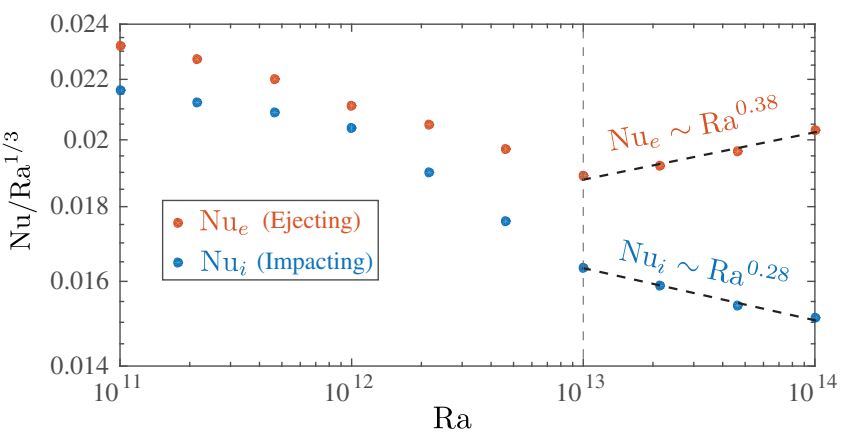

FIG. 4. Local wall-heat flux as a function of Ra, separately for the plume-ejecting region $\left(\mathrm{Nu}_{e}\right)$ and the plume-impacting region $\left(\mathrm{Nu}_{i}\right)$. At $\mathrm{Ra}^{*}=10^{13}, \mathrm{Nu}_{e}$ starts to undergo a transition to the ultimate regime with an effective scaling exponent of 0.38 , while $\mathrm{Nu}_{i}(\mathrm{Ra})$ has a much smaller effective scaling exponent of 0.28 .

$\beta_{e}<1 / 3$. However, beyond $\mathrm{Ra}^{*}, \mathrm{Nu}_{i}$ and $\mathrm{Nu}_{e}$ diverge. The ejecting regions show an increased heat transport, with $\beta_{e}=0.38$, which is precisely the ultimate scaling exponent predicted for $\mathrm{Ra} \sim \mathcal{O}\left(10^{14}\right)$ with logarithmic BLs. In contrast, the impacting regions have a much lower scaling exponent $\beta_{i}=0.28$. This means that the flow is partially in the ultimate regime and partially still in the classical regime. Based on these, we express the global Nusselt number, $\mathrm{Nu}=\mathrm{Nu}_{i}+\mathrm{Nu}_{e}$, in analogy to the GrossmannLohse approach [10,12], wherein the dissipation rate was separated into bulk and BL contributions. We write $\mathrm{Nu}=C_{i} \mathrm{Ra}^{\beta_{i}}+C_{e} \mathrm{Ra}^{\beta_{e}}$, where $\beta_{e}$ is expected to become even larger with increasing $\mathrm{Ra}$ [12]. The above expression asymptotically approaches the ultimate regime scaling when the plume-ejecting regions become more and more dominant in transporting the heat with increasing Ra. Thus, with only the local temperature profile being logarithmic (in plume-ejecting regions), the system can still undergo a gradual transition to the ultimate regime.

Finally, it is worthwhile to clarify the effect of the imposed two-dimensionality on the heat transfer. As mentioned in the beginning, $2 \mathrm{D} \mathrm{RB}$ is different from 3D RB. However, the effective scaling exponents observed are identical in 2D and 3D for a wide range of $\mathrm{Ra}$ in the classical regime [27], and here we found that also in 2D the transition starts at $\mathrm{Ra}^{*}=10^{13}$. Furthermore, the logarithmic BLs are theoretically expected for both $2 \mathrm{D}$ and $3 \mathrm{D}$, as the theoretical argument [13] is built on the Prandtl equations, which are 2D. Also in other 2D canonical flows, logarithmic BLs have been observed, e.g., in channel flow $[44,45]$. Therefore, the physical insights gained from this work are useful for understanding the transition to ultimate turbulence in both 2D and 3D flows.

In conclusion, we have used two-dimensional simulations of Rayleigh-Bénard convection to investigate the transition to the ultimate regime of thermal convection. We followed the approach of using the local flow structures to explain the globally observed heat transfer enhancement. 
A transitional Rayleigh number $\mathrm{Ra}^{*}=10^{13}$ was found for the $2 \mathrm{D} \mathrm{RB}$ with $\operatorname{Pr}=1$, beyond which the mean velocity profile has a log layer spanning one decade. However, the temperature profile is logarithmic only within the regions where plumes are ejected. The local effective Nusselt scaling exponent $\beta_{e}$ increases to 0.38 in the plume-ejecting regions, corresponding to the ultimate regime. The transition to the ultimate regime can be understood as the gradual takeover of the global heat transport by the contribution from the regions of plume ejection. In future work, we will extend these 2D DNS to smaller (and larger) Pr, to check the predicted Pr dependence $[10,11]$ of the transition to the ultimate regime.

Many open questions remain, for example whether wall roughness can trigger a transition to an asymptotic ultimate regime, in which $\mathrm{Nu} \sim \mathrm{Ra}^{1 / 2}$, i.e., the logarithmic corrections vanish. A previous study that reached $\mathrm{Ra}=10^{12}$ has shown that this was not yet the case [46]. However, in rough wall Taylor-Couette (TC) simulations (reaching a Taylor number of $\mathrm{Ta} \approx 2 \times 10^{9}$ ) and experiments (reaching $\mathrm{Ta} \approx 10^{12}$ ) we did reach the corresponding asymptotic ultimate regime for the angular momentum transport in TC flow thanks to the effect of pressure drag [47]. As the analog to pressure drag is absent in the heat flux balance for RB flow, such an asymptotic ultimate regime may not exist in RB flow [48].

We thank Daniel Chung for discussions and for pointing us to Ref. [48]. The work was financially supported by NWO-I, NWO-TTW, the Netherlands Center for Multiscale Catalytic Energy Conversion (MCEC), all sponsored by the Netherlands Organization for Scientific Research (NWO), and the COST Action MP1305. Part of the simulations were carried out on the Dutch national e-infrastructure with the support of SURF Cooperative. We also acknowledge PRACE for awarding us access to Marconi based in Italy at CINECA under PRACE Project No. 2016143351 and the DECI resource Archer based in the United Kingdom at Edinburgh with support from the PRACE aisbl under Project No. 13DECI0246.

*xiaojue.zhu@utwente.nl

†d.lohse@utwente.nl

[1] G. Ahlers, S. Grossmann, and D. Lohse, Rev. Mod. Phys. 81, 503 (2009).

[2] D. Lohse and K.-Q. Xia, Annu. Rev. Fluid Mech. 42, 335 (2010).

[3] F. Chilla and J. Schumacher, Eur. Phys. J. E 35, 58 (2012).

[4] C. Sun, Y. H. Cheung, and K.-Q. Xia, J. Fluid Mech. 605, 79 (2008).

[5] Q. Zhou and K.-Q. Xia, Phys. Rev. Lett. 104, 104301 (2010).

[6] Q. Zhou, R. J. A. M. Stevens, K. Sugiyama, S. Grossmann, D. Lohse, and K.-Q. Xia, J. Fluid Mech. 664, 297 (2010).

[7] R. du Puits and C. Willert, Phys. Fluids 28, 044108 (2016).

[8] R. H. Kraichnan, Phys. Fluids 5, 1374 (1962).
[9] E. A. Spiegel, Annu. Rev. Astron. Astrophys. 9, 323 (1971).

[10] S. Grossmann and D. Lohse, J. Fluid Mech. 407, 27 (2000).

[11] S. Grossmann and D. Lohse, Phys. Rev. Lett. 86, 3316 (2001).

[12] S. Grossmann and D. Lohse, Phys. Fluids 23, 045108 (2011).

[13] S. Grossmann and D. Lohse, Phys. Fluids 24, 125103 (2012).

[14] R. J. A. M. Stevens, E. P. van der Poel, S. Grossmann, and D. Lohse, J. Fluid Mech. 730, 295 (2013).

[15] M. V. R. Malkus, Proc. R. Soc. A 225, 196 (1954).

[16] C. H. B. Priestley, Aust. J. Phys. 7, 176 (1954).

[17] J. Niemela and K. R. Sreenivasan, New J. Phys. 12, 115002 (2010).

[18] P. Urban, P. Hanzelka, V. Musilová, T. Králík, M. La Mantia, A. Srnka, and L. Skrbek, New J. Phys. 16, 053042 (2014).

[19] X. Chavanne, F. Chilla, B. Castaing, B. Hebral, B. Chabaud, and J. Chaussy, Phys. Rev. Lett. 79, 3648 (1997).

[20] X. Chavanne, F. Chilla, B. Chabaud, B. Castaing, and B. Hebral, Phys. Fluids 13, 1300 (2001).

[21] X. He, D. Funfschilling, H. Nobach, E. Bodenschatz, and G. Ahlers, Phys. Rev. Lett. 108, 024502 (2012).

[22] X. He, D. Funfschilling, E. Bodenschatz, and G. Ahlers, New J. Phys. 14, 063030 (2012).

[23] R. J. A. M. Stevens, D. Lohse, and R. Verzicco, J. Fluid Mech. 688, 31 (2011).

[24] O. Shishkina, R. J. A. M. Stevens, S. Grossmann, and D. Lohse, New J. Phys. 12, 075022 (2010).

[25] R. J. A. M. Stevens, R. Verzicco, and D. Lohse, J. Fluid Mech. 643, 495 (2010).

[26] J. Schmalzl, M. Breuer, S. Wessling, and U. Hansen, Europhys. Lett. 67, 390 (2004).

[27] E. P. van der Poel, R. J. A. M. Stevens, and D. Lohse, J. Fluid Mech. 736, 177 (2013).

[28] Y.-X. Huang and Q. Zhou, J. Fluid Mech. 737 (2013).

[29] Y. Zhang, Y.-X. Huang, N. Jiang, Y.-L. Liu, Z.-M. Lu, X. Qiu, and Q. Zhou, Phys. Rev. E 96, 023105 (2017).

[30] D. R. Hewitt, J. A. Neufeld, and J. R. Lister, Phys. Rev. Lett. 108, 224503 (2012).

[31] G. Ahlers, E. Bodenschatz, D. Funfschilling, S. Grossmann, X. He, D. Lohse, R. J. A. M. Stevens, and R. Verzicco, Phys. Rev. Lett. 109, 114501 (2012).

[32] G. Ahlers, E. Bodenschatz, and X. He, J. Fluid Mech. 758 , 436 (2014).

[33] E. P. van der Poel, R. Ostilla-Mónico, R. Verzicco, S. Grossmann, and D. Lohse, Phys. Rev. Lett. 115, 154501 (2015).

[34] A. E. Perry and M.S. Chong, J. Fluid Mech. 119, 173 (1982).

[35] I. Marusic, B. J. McKeon, P. A. Monkewitz, H. M. Nagib, A. J. Smits, and K. R. Sreenivasan, Phys. Fluids 22, 065103 (2010).

[36] A. J. Smits, B. J. McKeon, and I. Marusic, Annu. Rev. Fluid Mech. 43, 353 (2011).

[37] R. Verzicco and P. Orlandi, J. Comput. Phys. 123, 402 (1996).

[38] E. P. van der Poel, R. Ostilla-Mónico, J. Donners, and R. Verzicco, Comput. Fluids 116, 10 (2015).

[39] H. Johnston and C. R. Doering, Phys. Rev. Lett. 102, 064501 (2009). 
[40] See Supplemental Material at http://link.aps.org/ supplemental/10.1103/PhysRevLett.120.144502 for numerical details and movies.

[41] E. P. van der Poel, R. Ostilla-Mónico, R. Verzicco, and D. Lohse, Phys. Rev. E 90, 013017 (2014).

[42] S. B. Pope, Turbulent Flow (Cambridge University Press, Cambridge, England, 2000).

[43] A. M. Yaglom, Annu. Rev. Fluid Mech. 11, 505 (1979).

[44] V. S. L'vov, I. Procaccia, and O. Rudenko, Phys. Rev. E 79, 045304 (2009).
[45] D. Samanta, F. Ingremeau, R. Cerbus, T. Tran, W. I. Goldburg, P. Chakraborty, and H. Kellay, Phys. Rev. Lett. 113, 024504 (2014).

[46] X. Zhu, R. J. A. M. Stevens, R. Verzicco, and D. Lohse, Phys. Rev. Lett. 119, 154501 (2017).

[47] X. Zhu, R. A. Verschoof, D. Bakhuis, S. G. Huisman, R. Verzicco, C. Sun, and D. Lohse, Nat. Phys., DOI: 10.1038/ s41567-017-0026-3 (2018).

[48] P. R. Owen and W. R. Thomson, J. Fluid Mech. 15, 321 (1963). 